Simone Barco e Francesca Marra*

Università per Stranieri di Siena

https://doi.org/10.18778/8220-506-0.07

\title{
WE COME FROM NAPOLI: IL DIALETTO NELLE CANZONI DI LIBERATO COME TRATTO IDENTITARIO
}

Riassunto: Nella percezione dell'italianità all'estero, la musica da sempre gioca un ruolo rilevante. La tradizione musicale del Bel Paese è sempre stata fortemente connotata da un punto di vista diatopico, restando in costante dialogo con i dialetti che convivono con la lingua standard. Nell'ultimo periodo, in particolare, la nuova scena cantautoriale e alternativa si è progressivamente aperta all'uso di dialettalismi e regionalismi oltre che di gergalismi. In questo capitolo analizzeremo nello specifico l'uso che del dialetto napoletano fa nelle sue canzoni Liberato, il rapper partenopeo che in soli due anni ha legato il suo successo nazionale - oltre che alle sperimentazioni musicali e a un'oculata operazione di marketing e immagine che punta a preservarne l'anonimato - anche all'uso di mescolare nei suoi testi varie lingue, in particolare dialetto e inglese. Il contributo mira ad analizzare alcuni tratti della lingua utilizzata dal cantante, con particolare riferimento al rapporto tra l'uso del gergo giovanile e il riferimento agli stilemi e alle tematiche tipici della canzone napoletana, attraverso una riflessione sulle espressioni selezionate dall'autore. Ci chiederemo, infine, in che modo l'idioletto del cantante influisca sulla diffusione di una precisa immagine identitaria.

Parole chiave: linguistica della canzone, dialetto, napoletano, identità, plurilinguismo, rap.

Abstract: We come from Napoli: the dialect in Liberato's songs as an identity
marker. In the global public perception, Italian culture has always been strongly
associated with music. Traditionally, Italian song lyrics have been characterized by
a high diatopic variation, which means that authors tend to mix dialects and Italian
in their songs. In the last few years the contemporary music setting, and particularly
the independent scene, opened up to dialectal features, colloquialisms and slang. In
the present paper, we will discuss the use of Neapolitan by Liberato, a rapper who

* Pur essendo frutto di un lavoro comune, Simone Barco ha curato i paragrafi 1, 5, 6; Francesca Marra i paragrafi 2, 3, 4. 
achieved resounding success in Italy and abroad during the last two years through his musical experimentation, a smart marketing strategy and by concealing his identity to the public. The aim of this essay is to analyze Liberato's lyrics in order to evaluate whether the dialect he uses reflects the contemporary Neapolitan youth slang. In addition, we will investigate whether the mixture of languages in Liberato points to propagate a clear cut identity or not.

Keywords: song lyrics, dialect, Neapolitan, identity, polylanguaging, rap.

\section{Introduzione}

La canzone ha da sempre giocato un ruolo fondamentale nell'esportazione del concetto di italianità all'estero (Serianni 2011; Telve 2012; Bonomi e Coletti 2015), anche in virtù della presunta musicalità della lingua italiana ${ }^{1}$. Sin dal melodramma seicentesco, il panorama musicale internazionale si giova del contributo di opere e lessico italiani (Bonomi 2015). A fine Ottocento è tuttavia la canzone in dialetto il principale vettore della musica italiana all'estero (Telve 2012; Coveri 2019). La canzone napoletana, in particolare, "per la compiutezza della forma strofa-ritornello, la straordinaria efficacia narrativa, il melodizzare fresco di reminiscenze della tradizione operistica" costituisce un modello, "un vero e proprio canone per molti dei repertori $[\ldots]$ che si sarebbero formati in seguito in altre parti del mondo" (Fabbri 2001: 563-564). Attraverso la musica leggera, nella percezione estera il dialetto napoletano è finito spesso per essere "identificat[o] con l'italiano stesso" (Bonomi e Coletti 2015: 9), come dimostra il caso, tra tutti, di O Sole Mio (Capurro e Di Capua 1898), brano che, pur composto in dialetto napoletano, è nell'immaginario collettivo simbolo privilegiato di italianità, "a riprova del fatto che la culla della tradizione musicale italiana sia la città partenopea” (Coveri 2015: 127). La canzone melodica dialettale continua a godere di prestigio e di vita autonoma rispetto a quella italiana fin quando, negli anni Settanta, le due strade vengono a intersecarsi, con lo sviluppo in contemporanea della canzone dialettale cantautoriale, con Crêuza de mä di Fabrizio De André2 , e di quella underground ${ }^{3}$, con Sud Sound

1 Circa lo stereotipo della musicalità della lingua italiana. Cfr. Trifone, Giovanardi (2012); Bonomi, Coletti (2015); Trifone (2019).

2 Pubblicato nel 1984, è preceduto almeno dagli album di Pino Daniele che, a partire dal 1977, tra napoletano, italiano e inglese, fondono cantautorato e blues.

3 In questo periodo "il dialetto comincia ad assumere una connotazione ideologica (contro la banalizzazione linguistica favorita dall'azione omologatrice dei mass media) e questo accade soprattutto nei generi musicali meno legati alla nostra tradizione melodica, come il rap e il reggae" (Serianni e Antonelli 2011: 72). 
System, 99 Posse, Mau Mau (Coveri 2011; Sottile 2013, 2018). La musica napoletana, avendo partecipato a questi cambiamenti in tutte le sue declinazioni - dal cantautorato a base melodica (Enzo Gragnaniello), al jazz e blues (James Senese, Enzo Avitabile), passando per il folk-rock (Nuova Compagnia di Canto Popolare), l'hip hop, il reggae e la dub (99 Posse, Almamegretta, 24 Grana) - continua ancora oggi a rinverdire il panorama musicale nazionale spaziando dal cantautorato all'hip-hop, con artisti come Foja, Gnut, Giovanni Block, Clementino, per citare solo i più giovani.

In questo capitolo presenteremo in prospettiva dialettologica e sociolinguistica il recente caso di un giovane artista campano che, sfruttando le potenzialità del plurilinguismo a base dialettale, è riuscito a raggiungere un successo europeo, contribuendo con la sua musica alla percezione dell'italiano all'estero nell'ambito hip hop e trap: Liberato. La sua storia comincia il 13 febbraio 2017 con la pubblicazione, sull'omonimo canale YouTube, del video musicale Nove Maggio ${ }^{4}$. Quest'unico singolo ha richiamato quasi subito l'attenzione della stampa online ${ }^{5}$, generando interesse sempre crescente nel pubblico. Da allora al 9 maggio 2019 - data divenuta iconica per i fan del cantante $^{6}$ - sono state pubblicate 12 canzoni, ma non è trapelata alcuna informazione circa l'identità dell'autore, cosa che ha contribuito alla creazione di un caso mediatico ${ }^{7}$. Al di là della mera curiosità e oltre ogni operazione di marketing, ciò che affascina di Liberato è la sua capacità di coniugare diversi stili musicali (dall'hip-hop al melodico, dall'elettronica alla trap, dal reggaeton alla drum'n'bass) con una lingua ampiamente recettiva che, attraverso dialetto, gergo giovanile e plurilinguismo, sembra essere sempre tesa alla ricerca di autenticità ${ }^{8}$.

4 www.rollingstone.it/musica/news-musica/liberato-in-anteprima-il-video-dinove-maggio/351505/ [28/02/2020].

5 napoli.repubblica.it/commenti/2018/05/09/news/liberato_l_amore_non ha_volto-195895837/ [29/02/2020], www.internazionale.it/bloc-notes/giovanniansaldo/2019/05/11/liberato-album-recensione [29/02/2020].

6 Il primo concerto a Napoli nel 2018, così come la pubblicazione del suo primo album nel 2019, sono avvenuti il nove maggio.

$7 \mathrm{Al} 29$ febbraio 2020 Tu t'e scurdat' 'e me ha realizzato 20.596 .685 visualizzazioni su YouTube (youtu.be/AWQcDlHoE4o).

$8 \mathrm{Nel}$ presente intervento verranno presi in considerazione gli 11 brani contenuti nell'album omonimo rilasciato il 9 maggio 2019. Né nel formato fisico del disco, né sul web sono presenti i testi ufficiali delle canzoni (Antonelli 2010 avverte circa le problematicità dell'affidarsi ai testi spontaneamente riportati dagli utenti di internet), perciò questi sono stati trascritti secondo una trascrizione fonetica larga in base all'ascolto delle canzoni. 


\section{La Napoli di Liberato: innovazione e tradizione}

Dai testi di Liberato emerge chiaramente una volontà di aderenza alla realtà giovanile, esplicitata a livello tematico mediante il riferimento costante al gruppo di pari (1) e a pratiche e abitudini comuni (2):

(1) cu'e cumpagna tuoja mmiez' a ggenta

con i tuoi amici tra la gente

steva 'na bbomba cu'e cumpagna mia'

stavo alla grande con i miei amici

(2) nu ppocha r'indica pa nun panza'

un po' d'indica per non pensare

fa cartin' effilter

prepara una canna

fa nu tira tu, i nun ma fira

fai un tiro tu, a me non va

picceré si cchiù bbella 'ra weed

piccola, sei più bella dell'erba

Questa tensione verso la realtà giovanile trapela anche a livello linguistico. Il cantante infatti fa un uso innovativo del dialetto, attingendo al serbatoio del gergo giovanile, di cui sono prova numerose espressioni, idiolettali (3) o d'uso comune (4). Particolarmente interessanti sono gli esempi in (3), che rivelano la capacità dell'autore di rielaborare materiali linguistici tratti dal registro colloquiale e per questo in un certo senso usurati, riposizionandoli entro collocazioni inusuali. Così, un verbo scarsamente poetico come 'ngatastà (it. incastrare) viene ad assumere nuova forza espressiva se accostato ai sospiri della tradizione amorosa.

(3) m'e 'ngatastata rind' a nu sufpira

mi hai incastrato in un sospiro

c'a piogg' rint' 'o cor

con la pioggia nel cuore

9 Da notare qui un caso di dialettizzazione dell'italiano mio: nonostante la sintassi resti quella del napoletano, con la posizione postnominale del possessivo (v. par. 4), qui, alla forma napoletana mieja, viene preferita la forma mì, ricalcata sull'italiano mio con indebolimento della finale. 


\author{
ma ffunna ll'anama \\ mi sfondi l'anima \\ m'e sciarmat' 'o coro \\ mi hai straziato il cuore \\ m'e 'ppicciat' 'o cora \\ mi hai infiammato il cuore \\ ma so' 'mbriacat' 'e te ${ }^{10}$ \\ mi sono ubriacato di te \\ (4) ca so' rimmast' 'a sotta \\ ci sono rimasto sotto \\ ma staja appennenna \\ mi stai lasciando \\ stonga tutt' I love you \\ sono innamorato / sono strafatto \\ tutt' 'o bblocc' \\ in fin dei conti
}

All'uso del dialetto entro il tessuto della canzone sono state attribuite due funzioni primarie: quella endolinguistica che lo vede come serbatoio di soluzioni metrico-ritmiche e quella extralinguistica che lo intende come manifesto ideologico (Coveri 1996). Mentre la prima soluzione è generalmente collegata alla musica cantautoriale, la seconda lo è al filone neodialettale; in accordo con quanto sostiene Sottile $(2013,2018)$, però, questa distinzione, seppur valida in via preliminare, non è facilmente applicabile al caso singolo. In tal senso, la presenza in Liberato di una commistione di elementi tradizionali da un lato ed innovativi dall'altro, tanto da un punto di vista linguistico che tematico, suggerisce la possibilità che il dialetto, usato coscientemente dai giovani, possa assumere il carattere di lingua viva, seppure in dialogo costante con il proprio passato ${ }^{11}$.

10 Che rimanda a Me so' mbriacato di Mannarino (2009).

11 "Quest'omaggio ai tempi antichi della parola dialettale [...] finisce per svelare usi giovanili consapevoli e sorvegliati del dialetto che impongono una riflessione sul rapporto tra la straordinaria capacità di parlanti giovani di riusare artisticamente materiale linguistico-culturale dato per spacciato, e la straordinaria resistenza di questo universo alle scosse provocate dalla modernità e dalla globalizzazione" (Sottile 2013: 78). 
In particolare, il riferimento all'immaginario culturale partenopeo assume in Liberato la duplice forma della citazione puntuale e dell'allusione sottesa. Mentre la seconda si evince da fattori quali il ritorno costante del mese di maggio ${ }^{12}$ o l'appropriazione di concetti cari alla collettività, come quello di appucundria ${ }^{13}$, la prima costituisce una strategia estremamente produttiva e costante nella costruzione del testo $(5)$, secondo un uso già caro al cantautorato napoletano (Avolio 2015) ${ }^{14}$ :

(5) t'e pigliat' 'a vita mia (Je te voglio bene assaje) t’aje pigliato 'a vita mia (Core 'ngrato, Chiaramello e Cardello 1911)

rint' a 'sta bufia pierd' 'o suonn'e 'a fantasia (Je te voglio bene assaje) ch'aggio perduto 'o suonno e 'a fantasia (Dicitencello vuje, Fusco e Falvo 1930)

passann' e fpassanna sott' a 'stu bbalcona (Nunn a voglio ncuntrà) e passe e spasse sott’a stu balcone (Guaglione, Salerno e Fanciulli 1956)

so' rimmast' sott' a bbott' 'mbressiunata (Nove maggio)

e' 'a femmena è restata sott'a botta, 'mpressiunata

(Tammurriata nera, Mario e Nicolardi 1944)

Ma Liberato si spinge oltre la tendenza citazionistica, onorando la tradizione anche e soprattutto attraverso il ricorso ai topoi del temario amoroso: l'amore tormentato, non corrisposto, tradito, rappresenta il nucleo tematico maggiormente sviluppato dall'autore (6).

(6) m'arrevuot' 'o cora e ppoi ta na vaja

mi stravolgi il cuore e poi vai via

picceré ma faja asci pazza

piccola mi fai impazzire

stai cu issa ma po' chiamma ccà

stai con lui e poi chiami me

12 Noto alla canzone napoletana sin da Era de maggio (Di Giacomo e Costa 1885), Na sera e maggio (Cioffi, Pisano 1937).

13 www.treccani.it/magazine/lingua_italiana/articoli/parole/Daniele.html [29/02/2020]. Il lessema, reso famoso dall'omonima canzone di Pino Daniele, ritorna in Gaiola portafortuna.

14 Pino Daniele, tra gli altri, "si mostra [...] particolarmente abile nell'inserire, all'interno dei testi, frasi ed espressioni assai comuni, tratte direttamente dal patrimonio dialettale collettivo, dalla tradizione più condivisa, quasi a voler dare un tocco inconfondibile e rassicurante di 'quotidianità', frammenti in cui chiunque sia originario della zona possa riconoscersi” (Avolio 2015: 61). 


\section{La Napoli di Liberato: geografia}

Il legame con Napoli trapela, infine, dalla creazione di una vera e propria geografia della canzone che consente, facendo leva sull'immaginario popolare collettivo, di promuoverne anchei contenuti, secondo una pratica molto comune in ambito musicale ${ }^{15}$. È così che, in Liberato come nel rap metropolitano, "anche l'odonomastica diventa costitutiva dei testi musicali" (Sottile 2013: 63), e Mergellina, Procida, Marechiaro si trasformano in tasselli di una realtà in cui il fruitore riesce a districarsi e, di conseguenza, riconoscersi (7):

(7) scannimm' a Mergellina andiamo a Mergellina

chiova 'ngopp' a Procida piove su Procida

chiova 'ngopp' a Nisida

piove su Nisida

nu ggir' a Marechiaro

un giro a Marechiaro

na fest' a Trentaremi

una festa a Trentaremi

tu ma vas' a Materdei

tu mi baci a Materdei

'uard' 'e fuocha 'bbasca Forcella

guarda i fuochi su Forcella

Come nota Sottile (2013: 67) in riferimento alla realtà siciliana, "una scelta linguistica diatopicamente marcata $[\ldots]$ pare $[\ldots]$ favorire la composizione di canzoni che presentino nel testo o nel titolo quella terra di cui il dialetto è il codice tradizionale": terra e dialetto si intrecciano, quindi, al fine di costruire uno scenario ben definito, in cui sia facile riconoscere il capoluogo campano con le abitudini, i suoni, i personaggi che lo popolano.

15 "La consuetudine di comporre canzoni sfruttando gli elementi toponimici tradizionali è abbastanza diffusa e costituisce uno specifico modo di attingere al serbatoio della cultura popolare per riproporne parte dei contenuti" (Sottile 2013:61). 


\section{Il dialetto di Liberato}

Il dialetto di Liberato presenta i tratti salienti del napoletano ${ }^{16}$. Tra quelli più rilevanti, troviamo abitualmente sia fenomeni fonetici, quali metafonia (miso; fafiva; sapiva; stiva ${ }^{17}$, raddoppiamento fonosintattico (s'éffatta; e ppo'; cu' tte $)^{18}$, raddoppiamento fonosintattico del genere neutro ('nu ppocha), gammacismo ('uappə; 'uardəmə), betacismo (chilli vasa), rotacismo (a lufe r'o mara; nu ppocha r' indica; parta rimmanə; 'na vota rifista; rifamə; rammə) $)^{19}$, sonorizzazione dell'occlusiva in contesto postnasalico ('ngatastatz; rinda; 'n'gapa; quanda; semba), che fenomeni morfo-sintattici, quali inversione dell'ordine canonico aggettivo possessivo-nome nel sintagma nominale non marcato ('a vita mia; 'a casa mia; 'o cora mio; 'e cumpagna mì), risalita del clitico rispetto all'infinito (J'alluntanà; po to fcurdà; po sə 'nnammurà; po nun to sunnà; po to fa turnà; to 'ncuntrà) e al modale (nunn 'a voglio 'ncuntrà), posposizione dell'avverbio focalizzante (i to voglio bene assaja; 'na vota sola ancora; 'n'ata vota ancora).

Il dialetto sembra, dunque, lingua nativa dell'autore, non soltanto alla luce della scelta compositiva operata, ma anche perché del napoletano rispetta le constraints fonetiche e morfo-sintattiche. Si può notare inoltre che, laddove il cantante si volge all'uso dell'italiano, fa ricorso al proprio italiano regionale. Questo è quanto accade laddove si ha una pronuncia geminata delle scempie italiane in posizione intervocalica (8), nonché nei casi di palatalizzazione della fricativa davanti a occlusiva velare sorda $/ \mathrm{k} /$ in italiano (9):

(8) ramma'na possi[bb]ilità

dammi una possibilità

Li[bb]erato

(9) non ti $[j k]$ ordar dime

16 Il napoletano, dialetto primario (primärer Dialekt) nella distinzione operata da Coseriu (1980), rientra fra i dialetti italiani centro-meridionali. Per una sua definizione si rimanda a Avolio (1995); De Blasi (2006); Ledgeway (2009); Loporcaro (2009).

17 Fenomeno di armonia vocalica riguardante le sillabe toniche e definibile come fenomeno di innalzamento delle vocali medio-alte /e/ ed /o/ per effetto di vocali finali alte /i/ ed /u/, come accade in napoletano per russa da lat. ROSSUM e rossa da lat. ROSSAM (cfr. Ledgeway 2009).

18 Fenomeno di rafforzamento consonantico dell'iniziale di parola per effetto della parola che precede, quando questa recava in latino consonante finale (cfr. Fanciullo 2015).

19 Fenomeni di indebolimento consonantico in contesto intervocalico per i quali le occlusive sonore velare /g/, labiale /b/ e dentale /d/ si affievoliscono, e vengono prodotte come fricative, fino a cadere del tutto in alcuni casi (cfr. Ledgeway 2009). 
Al di là dell'aderenza ai pattern fonotattici del napoletano, tuttavia, un'attenta analisi linguistica rivela la presenza di alcuni tratti che potremmo definire devianti rispetto al sistema. Un caso di incongruenza morfo-sintattica è, ad esempio, il mancato accordo di numero fra i verbi di due proposizioni:

(10) quanna stiva cu mmika

[E] ra na femmana senza poesia

quando stavi con me eri una donna senza poesia

In (10) l'imperfetto stiva della proposizione temporale introdotta da quanna, presentando l'esito metafonetico della vocale tonica, non può che essere una seconda persona singolare del verbo stare. Ci aspetteremmo, pertanto, di ritrovare anche nella principale una seconda persona singolare. Invece, qui troviamo la terza persona singolare dell'imperfetto di essere, $[\varepsilon] r$, priva di esito metafonetico, in luogo dell'atteso ira. Si tenga conto, tuttavia, del fatto che quanna stiva cu mmika è stilema preso dalla tradizione napoletana (Reginella, Bovio/Lama, 1917). Così, il mancato accordo di numero fra i verbi delle due proposizioni si spiegherebbe in virtù della già evidenziata tendenza citazionistica di Liberato. A favore di questa interpretazione, inoltre, gioca la presenza, in un altro punto della medesima canzone, del verso corretto quanna steva cu mme, recante esito metafonetico e opportunamente accordato ${ }^{20}$.

Tra le incongruenze fonetiche, invece, notiamo la saltuaria confusione tra vocali posteriori medio-basse e medio-alte:

(11) 'mmiez' a tuttr 'stu cazz' 'e baillamme ca stann ddoja [roso], teng' 'a capa gloriosa nanné, rimana ta [ jposa]

(12) ca verimma rimman' a Amsterdàm a'rò staja 'nna[jko]sə, mo' nunn è $[k k s] s a$

(13) 'ng[o]pp' 'o golfa

Sia in $\int p \jmath s ə(11)$ che in ' $n n a[j k \jmath] s a$ (12) la presenza della vocale posteriore medio-bassa / $\mathrm{o}$ suscita qualche dubbio; in napoletano ci aspetteremmo, infatti, di trovare in queste sedi una vocale posteriore medio-alta /o/. Siamo, dunque,

20 In questo stessa coppia di versi, poi, è da notare che il pronome comitativo MECǓM > mika, ormai percepito come arcaico, viene sostituito dal più attuale me. È da segnalare, infine, che era è anche forma della seconda persona singolare dell'italiano regionale, per cui potrebbe trattarsi di un caso di interferenza fra dialetto e italiano regionale. 
portati a interpretare questa sostituzione come l'esito di esigenze metriche,

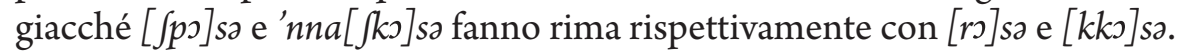

In (13) segnaliamo, invece, il processo inverso a quello sopra descritto, ovverol'innalzamento della vocale posteriore medio-bassa $/ \mathrm{\rho} /$ nella preposizione 'ngoppa (it. su, sopra). Riteniamo che tale resa vocalica sia qui imputabile alla posizione debole della sillaba in cui la vocale ricorre nella catena fonica del cantato; seppure sia accentata a livello di parola e pertanto passibile di un vocalismo eptavocalico, nella prosodia frasale la suddetta sillaba risulta debole e, probabilmente a causa della velocità del cantato, è pronunciata come chiusa.

Ancora, un tratto che rende riconoscibile il napoletano è l'innalzamento delle vocali medio-basse nel dittongo ascendente. Ciò significa che, laddove in italiano troveremmo dittonghi formati da approssimante + vocale mediobassa $^{21}$, in napoletano troviamo approssimante + vocale medio-alta. Per questo motivo, la presenza di vocali medio-basse nei dittonghi ascendenti in $f[w o]$ cha,

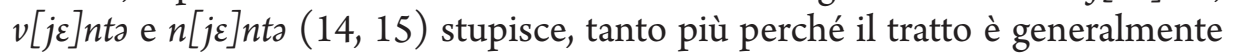
così radicato da imprimersi anche nella pronuncia dell'italiano regionale dei parlanti nativi. Si tratta, tuttavia, di un caso isolato per quanto concerne la vocale medio-bassa posteriore in (14) e per questo interpretabile come fenomeno eccezionale, e di casi innescati per assonanza da esigenze di rima in (15):

(14) 'uard' 'ef[wo]cho

(15) 'e pparola tuoja s'e pport'o $v[j \varepsilon]$ nta cu 'e cumpagna tuoja mmiez' a gg[E]nta stong' arrefunnenna malam $[\varepsilon]$ nta chiagna sanga, ma è ccos' 'e $n[j \varepsilon] n t a$

Esiti inattesi o devianti non devono indurci a giudizi affrettati circa l'effettiva competenza linguistica di Liberato, in quanto è noto che la lingua cantata sottostà a delle esigenze ritmico-stilistiche differenti rispetto al parlato, in ragione delle quali esiti simili si configurano semplicemente come possibilità compositive ulteriori per il cantante ${ }^{22}$.

$21 \mathrm{Ma}$ anche laddove il napoletano dittonga metafoneticamente in maniera autonoma rispetto all'italiano, come nel caso di $v[j e] n t z$ (it. $v[\varepsilon] n t o$ ).

22 È certo che "una canzone non è una canzone senza musica, e non lo è senza testo" (Cartago e Fabbri 2016: 257), per cui analizzando un testo bisognerebbe sempre tenere aperto il confronto con musica, metrica e stile, dal momento che "i versi stampati [...] non possono darci se non un'immagine parziale, molto riduttiva di un testo complesso, fatto di parole ma anche di suoni, di timbri, di gesti" (Fiori 1996: 146). Per il legame tra parole e musica cfr. Zuliani (2018). 
La radicata napoletanità di Liberato si evince, d'altronde, anche dalla presenza di fenomeni di transfer dalla L1 dell'artista alle L2 di cui si serve, come la pronuncia geminata della consonante intervocalica in inglese (16), la posteriorizzazione della fricativa interdentale sorda $/ \theta /$ resa come fricativa dentale sorda /s/ in posizione intervocalica e la caduta della consonante finale di parola in spagnolo (17), la presenza di raddoppiamento fonosintattico e la caduta della consonante finale di parola in inglese $(18,19)$ :

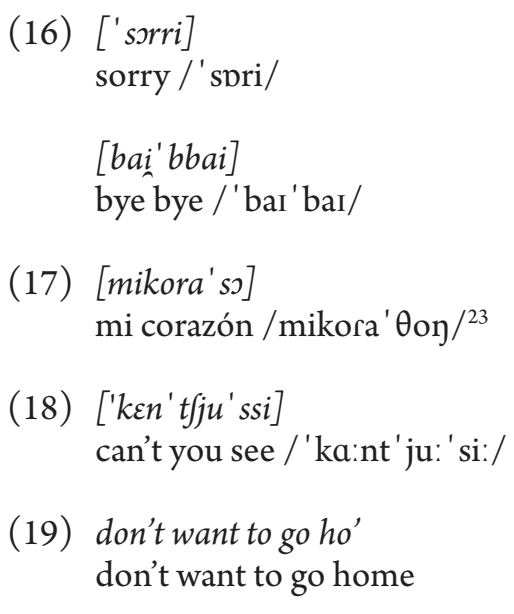

Di più difficile decodifica potrebbero risultare i fenomeni opposti, pure molto meno consistenti a livello quantitativo, ovvero i casi di realizzazione ipercorretta della pronuncia italiana, che ancora una volta però si spiegano in termini di libertà espressiva del cantante. Si è già detto della prototipicità della pronuncia chiusa del dittongo ascendente napoletano. Troviamo pertanto degno di nota il fatto che in (20) cuore rechi vocale posteriore medio-bassa $/ \mathrm{J} /$, secondo la corretta pronuncia italiana. Infine, l'assenza di neutralizzazione della vocale finale in (21), interpretabile come un caso di italianizzazione, ci sembra invece dipendere da esigenze metriche, in quanto rima con il successivo Odisseo.

(20) il cuore mi batteva

(21) si nun c'appiccicamma i' nun pareo ccà ddinta Scorr' 'o sanga r' Odisseo

23 Qui, chiaramente, non si può trascurare la possibilità che il cantante abbia come riferimento varianti di spagnolo in cui la fricativa interdentale sorda è sostituita dalla fricativa dentale sorda. 
Facendo dialogare tradizione e innovazione, giocando col dialetto, Liberato fa uso di un idioletto vivo e produttivo. La canzone d'amore è declinata al presente, la tradizione percorre suoni nuovi e nuove collocazioni e l'adesione idiomatica, musicale e stilistica dell'autore alla realtà giovanile determina la necessità di aprirsi ad altri mondi e dunque ad altre lingue.

\section{Dal dialetto alle altre lingue}

Nel terreno prettamente dialettale dei testi di Liberato, infatti, trovano spazio inserzioni mistilingui, soprattutto in inglese (22), ma anche in spagnolo (23), francese (24) e italiano (25):

(22) 'miez' 'a via allucc stand by me cə'ntussecamma, you'll never change

(23) tu labios c'a lufe r' 'o mar no puedo dejar de mirar

(24) Bye, bye, mon amour on s'appella après

appjennə 'tta cosə fuitennə cu' mme

(25) teng' 'o core sott'anestesia

Gli esempi di contatto nei testi di Liberato verranno visti da vicino in un'altra sede, qui basti segnalare che l'uso plurilinguistico - con una spiccata preferenza verso l'inglese - è caratteristica che innerva al punto le canzoni dell'autore da diventarne cifra stilistica distintiva e riconoscibile. Le altre lingue, tra le varie funzioni, aiutano a chiudere una rima (26), servono da allocutivo (27), richiamano alla mente un riferimento intertestuale (28), e, più in generale, completano la struttura metrica della canzone, fungendo per esempio da riempitivo e da refrain (29):

(26) baby, I'm so high e nun sacca cu' cchi staja

(27) mi princesa nun fa accussì

(28) Un giorno all'improvviso Mi innamorai di te 
Il cuore mi batteva

mo rifamə pacchée ${ }^{4}$

(29) 'e ccinga r'a matina, it's me and you

[...]

parlammə chianu chianə, it's me and you

Le maniere linguistiche di Liberato ricalcano l'uso giovanile del polylanguaging, presente soprattutto nella comunicazione via $\mathrm{web}^{25}$, che prevede "the use of features associated with different languages even when speakers know only few features associated with [them]" (Jørgensen et al. 2011: 33). Per quanto quest' uso sia generalmente legato a una dimensione ludica ${ }^{26}$, "non si può escludere che il polylanguaging svolga anche una funzione socio-identitaria: serve infatti a esprimere una identità multiforme e composita, dove accanto [...] alle componenti [...] locali, trovano posto componenti cosmopolite e globalizzate" (Alfonzetti 2013: 245). Liberato sembra in effetti fare un uso cosciente tanto del plurilinguismo quanto del dialetto stesso: la lingua gioca un ruolo fondamentale nella sua opera così come nella costruzione del suo personaggio. Il dialetto, infatti, diventa anche lingua della comunicazione del cantante col pubblico ${ }^{27}$, lingua veicolare dei social ${ }^{28}$ e del merchandising ${ }^{29}$. Allo stesso modo, le altre lingue contribuiscono all'iconicità dei versi e di espressioni diventate di successo nel pubblico. Liberato sembra dirci che il napoletano ha tutte le possibilità linguistiche, metriche, funzionali, per prendere parte alla comunicazione odierna; per questo motivo l'italiano viene

24 Si tratta della citazione di un coro dei tifosi della Società Sportiva Calcio Napoli.

25 E sempre più attestato nel panorama hip-hop, soprattutto trap, come dimostrano, per citare solo alcuni, Ghali e Mahmood con l'arabo, Dark Polo Gang e Tha Supreme con l'inglese.

26 "Language users employ whatever linguistic features are at their disposal to achieve their communicative aims as best they can, regardless of how well they know the involved languages" (Jørgensen et al. 2011:34).

27 Nell'unica intervista rilasciata, il cantante risponde esclusivamente in napoletano alle domande poste in italiano, www.rollingstone.it/musica/ interviste-musica/liberato-abbiamo-intervistato-luomo-misterioso-dietro-novemaggio/357693/ [29/02/2020].

28 Il 10 febbraio 2020, un nuovo concerto viene annunciato con queste parole sul profilo ufficiale Instagram: "E bigliett' p'o 25 aprile so' fernùt' 'o ffacimm' n'ata vot' once again nuova data 26 aprile".

29 Per cui la sacca è 'a bborz', l'accendino griffato è o 'clippèr 'e fierr', liberato. bigcartel.com/. 
evitato $^{30}$, sostituito dall'inglese, che svolge quindi, nella canzone, le funzioni ausiliarie che, nel discorso spontaneo, sono proprie dell'italiano. Il dialetto può essere indipendente dalla "morsa" dell'italiano e, attraverso l'incontro con altre lingue, mantenere la sua fisicità e il suo fascino, difendendosi dall'esclusione dal processo di globalizzazione e anzi partecipandovi attivamente. Il dialetto locale, mostrando la propria capacità di parlare una lingua giovane, gioca un ruolo centrale, dunque, nella formazione di un'identità globale.

\section{Conclusioni. Identità, napoletanità, italianità}

Nel suo progetto di raccontare la realtà napoletana, Liberato opera a monte una scelta compositiva chiara, che è quella di favorire il dialetto a discapito dell'italiano, forse credendo che, essendo il dialetto una lingua al pari dell'italiano, da esso può (e quindi deve) essere indipendente. Per fare ciò, cerca di trincerarsi in (a) un dialetto "svecchiato", infarcito di espressioni prese dal gergo giovanile napoletano (esempi 1-4); (b) all'opposto, un dialetto letterario, ripescato dalla secolare tradizione musicale partenopea (esempi 5); (c) un plurilinguismo in cui l'inglese, lingua madre dell'hip hop, fa, in un certo senso, le veci dell'italiano (esempi 22-29). In questo modo sdogana l'uso del dialetto nel mondo globalizzato: il napoletano parla la lingua dei giovani, può esprimere autonomamente concetti di solito appannaggio dell'italiano, può competere "perfino" con l'inglese.

Tra le motivazioni che portano Liberato alla scelta del dialetto non mancano ragioni metrico-espressive, né simbolico-ideologiche (Coveri 1996) a carattere etnico più che di protesta (Sottile 2018), e la riscoperta della tradizione non porta ai risultati ai quali normalmente giungono "i neomelodici napoletani, molto legati alla tradizione, chiusi in un immaginario - e quasi sempre in un mercato - locale" (Antonelli 2010: 203). È però evidente che anche qui, come in altri autori dialettali, "il dialetto diventa anche un simbolo di riscoperta e di riappropriazione identitaria” (Sottile 2013: 39) e Liberato sembra difendere e portare avanti, in modo molto cosciente, una certa idea di Napoletanità. Tuttavia, pur costruendo l'immagine di un mondo a parte rispetto all'Italia ${ }^{31}$, i cui confini geografici vengono segnalati ripetutamente (cfr. par. 3), il "localismo" di Liberato riesce a raggiungere un pubblico nazionale e non solo, come dimostrano l'attenzione dedicatagli da Netflix e da Converse,

30 Comparendo quasi esclusivamente come prestito di necessità e quasi sempre adattato (cuffiettz; nun visualizzi cchiù).

31 Narrazione portata avanti anche grazie al racconto visivo dei video girati (a Napoli) da Francesco Lettieri che accompagnano ogni brano. 
la sua collaborazione con i britannici Gaika e 3D ${ }^{32}$, il suo concerto a Barcellona il 15 giugno 2018, e quindi il fatto che su 6 concerti totali, solo uno si è tenuto a Napoli. La partecipazione da parte di un pubblico non campano mostra come l'identità locale riesca a diventare motivo di riconoscimento anche per chi non vive la realtà da cui quell'identità è nata, trasformandosi in identità "panregionale", inglobando gli ascoltatori di ogni parte d'Italia, che, grazie alla full immersion multimodale (visiva attraverso i video girati a Napoli, linguistica, ma anche musicale, se pensiamo alla riproposizione di un genere popolare come la tammurriata presente in Nunn'a voglio ncuntrà), si immergono nella realtà napoletana, cominciano a riconoscerne le fattezze, la geografia, i suoni e le parole. La dimensione locale diventa quindi specchio di quella nazionale ${ }^{33}$, e la difesa della napoletanità, attuata paradossalmente anche attraversol'ingresso di altre lingue nel tessuto dialettale, porta, in un certo senso, alla (ri)scoperta di un'identità sovralocale, all'interno della quale ogni ascoltatore, napoletano e non, può sentirsi parte di una comunità, nella quale la napoletanità non è altro che un surrogato, o forse un sinonimo, di italianità.

\section{Bibliografia}

Alfonzetti, Giovanna (2013). "Il polylanguaging: una modalità di sopravvivenza del dialetto nei giovani”, Bollettino del Centro di studi filologici e linguistici siciliani, 24, pp. 213-252.

Antonelli, Giuseppe (2010). Ma cosa vuoi che sia una canzone. Mezzo secolo di italiano cantato, Bologna, il Mulino.

Avolio, Francesco (1995). Bommèspra. Profilo linguistico dell'Italia centro-meridionale, San Severo, Gerni Editori.

Avolio, Francesco (2015). “'O dialètt' r"o bblìs. Per un'analisi linguistica delle canzoni di Pino Daniele”, InVerbis, 2, pp. 51-70.

$32 \mathrm{Al}$ secolo Robert Del Naja, membro dei Massive Attack, già legato a Napoli oltre che dalle origini italiane, anche dalla partecipazione alla colonna sonora di Gomorra di Matteo Garrone nel 2008. Così il profilo Instagram di Liberato annuncia l'uscita del singolo: "Liberato canta ancora cu' 3D ('re Massive Attack) e Gaika".

33 A sottolinearlo è, per quanto riguarda l'aspetto visivo, il regista dei video di Liberato, Francesco Lettieri, il quale, a proposito della ricerca di universalità che rompa gli stereotipi locali, così si esprime: "Lapproccio scelto assieme a Liberato è stato quello di mettere ovunque il Vesuvio, la classica cartolina rappresentativa della città. Abbiamo scelto di giocare proprio nel rimarcare lo stereotipo che volevamo scardinare. Non vogliamo certo negare che Napoli sia anche i ragazzini in motorino, il Vesuvio, la pizza e il mandolino. Però se andiamo a guardare questi elementi da vicino diventano delle storie e dei personaggi al di là della provenienza geografica: napoletani o meno che siano quello che vogliamo raccontare potrebbe svolgersi in qualunque parte del mondo". xl.repubblica.it/articoli/francesco-lettieri-io-liberato-enapoli/70508/ [29/02/2020]. 
Berruto, Gaetano (2004). Prima lezione di sociolinguistica, Roma-Bari, Laterza.

Bonomi, Ilaria (2015). "Italianismi musicali nel mondo", [in] Ilaria Bonomi e Vittorio Coletti (a c. di), L'italiano della musica nel mondo, Firenze, Accademia della Crusca, pp. 10-30.

Bonomi, Ilaria e Coletti, Vittorio (2015). "Premessa", [in] Ilaria Bonomi e Vittorio Coletti (a c. di), L'italiano della musica nel mondo, Firenze, Accademia della Crusca, pp. 7-9.

Cartago, Gabriella e Fabbri, Franco (2016). "La lingua della canzone", [in] Ilaria Bonomi e Silvia Morgana (a c. di), La lingua italiana e i mass media, Roma, Carocci.

Coseriu, Eugeniu (1980). “'Historische Sprache' und 'Dialekt”, [in] Joachim Göschel, Pavle Ivić e Kurt Kehr (a c. di), Dialekt und Dialektologie, Steiner, Wiesbaden, pp. 106-122.

Coveri, Lorenzo (1996). "Per una storia linguistica della canzone italiana. Saggio introduttivo", [in] Lorenzo Coveri (a c. di), Parole in musica. Lingua e poesia nella canzone d'autore italiana. Saggi critici e antologia di testi di cantautori italiani, Novara, Interlinea.

Coveri, Lorenzo (2011). "Le canzoni che hanno fatto l'italiano", [in] Elisabetta Benucci e Raffaella Setti (a c. di), Italia linguistica: gli ultimi 150 anni. Nuovi soggetti, nuove voci, un nuovo immaginario, Firenze, Le Lettere, pp. 69-126.

Coveri, Lorenzo (2015). "Italiano formato export. Dieci canzoni italiane per il mondo", [in] Ilaria Bonomi e Vittorio Coletti (a c. di), L'italiano della musica nel mondo, Firenze, goWare, pp. 122-139.

Coveri, Lorenzo (2019). “L'italiano delle canzoni nel mondo”, [in] Carla Bagna e Laura Ricci (a c. di), Il mondo nell'italiano, l'italiano nel mondo, Pisa, Pacini, pp. 127-135.

De Blasi, Nicola (2006). Profilo linguistico della Campania, Roma-Bari, Laterza.

Fabbri, Franco (2001). "La canzone”, [in] Jean-Jacques Nattiez (a c. di), Enciclopedia della musica. Volume primo. Il Novecento, Einaudi, Torino, pp. 551-576.

Fanciullo, Franco (2015). Prima lezione di dialettologia, Roma-Bari, Laterza.

Fiori, Umberto (1996). “'In un supremo anelito'. L'idea di poesia nella canzone italiana”, [in] Rossana Dalmonte (a c. di), Analisi e canzoni, Trento, Dipartimento di Scienze Filologiche e Storiche, pp. 145-160.

Grassi, Corrado, Sobrero, Alberto A., Telmon, Tullio (2003). Introduzione alla dialettologia italiana, Roma-Bari, Laterza.

Jørgensen, J. Normann et al., (2011). "Polylanguaging in Superdiversity”, Diversities, 13 (2), pp. 23-37.

Ledgeway, Adam (2009). Grammatica diacronica del napoletano, Tübingen, Max Niemeyer Verlag.

Loporcaro, Michele (2009). Profilo linguistico dei dialetti italiani, Roma-Bari, Laterza.

Serianni, Luca (2011). "L'italiano nel mondo", [in] Vittorio Coletti (a c. di), L'italiano dalla nazione allo Stato, Firenze, Le Lettere, pp. 227-231.

Serianni, Luca e Antonelli, Giuseppe (2011). Manuale di linguistica italiana. Storia, attualità, grammatica, Milano, Bruno Mondadori.

Sottile, Roberto (2013). Il dialetto nella canzone italiana degli ultimi venti anni, Roma, Arcana.

Sottile, Roberto (2018). Dialetto e canzone. Uno sguardo sulla Sicilia di oggi, Firenze, Cesati.

Telve, Stefano (2012). That's amore! La lingua italiana nella musica leggera straniera, Bologna, il Mulino. 
Trifone, Pietro (2019). "Italiano lingua di cultura. Riabilitazione di un luogo comune", [in] Carla Bagna e Laura Ricci (a c. di), Il mondo nell'italiano, l'italiano nel mondo, Pisa, Pacini, pp. 99-106.

Trifone, Pietro e Giovanardi, Claudio (2012). L'italiano nel mondo, Roma, Carocci.

Zuliani, Luca (2018). L'italiano della canzone, Roma, Carocci.

\section{Sitografia}

liberato.bigcartel.com/ [29/02/2020].

napoli.repubblica.it/commenti/2018/05/09/news/liberato_1_amore_non_ha_ volto-195895837/ [29/02/2020].

www.internazionale.it/bloc-notes/giovanni-ansaldo/2019/05/11/liberato-album-recensione [29/02/2020].

www.rollingstone.it/musica/news-musica/liberato-in-anteprima-il-video-di-novemaggio/351-505/ [29/02/2020].

www.treccani.it/magazine/lingua_italiana/articoli/parole/Daniele.html [29/02/2020].

xl.repubblica.it/articoli/francesco-lettieri-io-liberato-e-napoli/70508/ [29/02/2020].

youtu.be/AWQcDlHoE4o [29/02/2020]. 